\title{
Fibroadenoma with an unexpected lobular carcinoma in situ: A case report and review of the literature
}

\author{
BIN HUA $^{1}$, JING-YONG XU ${ }^{1}$, LEI JIANG ${ }^{2}$ and ZHENG WANG $^{3}$ \\ Departments of ${ }^{1}$ General Surgery, ${ }^{2}$ Radiology and ${ }^{3}$ Pathology, \\ Beijing Hospital, The Ministry of Health, Beijing 100730, P.R. China
}

Received May 26, 2014; Accepted May 14, 2015

DOI: $10.3892 / \mathrm{ol} .2015 .3488$

\begin{abstract}
Fibroadenoma (FA) is the most common type of breast lesion in young female individuals. Although malignant transformation in FA is rare, there is evidence of an association with breast carcinoma, particularly in middle-aged females with associated risk factors, such as a strong family history and/or BRCA-1/2 mutations. The current study presents the case of a 44-year-old female exhibiting lobular carcinoma in situ (LCIS) arising within an FA. Over the previous 20 years, numerous lumps were detected in the patient's breasts, all of which were clinically diagnosed as FA and expectantly managed. One year prior to presentation, the patient's father was diagnosed with esophageal cancer, older sister with kidney cancer and the patient herself with a benign ovarian teratoma. The patient presented with one lump in each breast and monitoring was performed using ultrasonography (US). US detected an increase in the size of the two lumps and analysis of the images was used to propose a diagnosis of FA. Therefore, a lumpectomy was performed. Analysis of the frozen section indicated a diagnosis of FA in the right breast, however, a 5-mm LCIS was identified within the FA of the left breast. Subsequent to the identification of three additional lumps in the left breast, a left mastectomy was performed. During a five-year follow-up period, no local recurrence or contralateral breast carcinoma occurred. The present study showed that FAs containing foci of carcinoma in situ can be indistinguishable from benign lesions following imaging, therefore, despite the incidence of carcinoma within FA is estimated at $0.1-0.3 \%$, it should be considered a long-term risk factor for invasive breast
\end{abstract}

Correspondence to: Dr Bin Hua, Department of General Surgery, Beijing Hospital, The Ministry of Health, 1 Dahua, Beijing 100730, P.R. China

E-mail: huabinbjh@126.com

Abbreviations: FA, fibroadenoma; LCIS, lobular carcinoma in situ; DCIS, ductal carcinoma in situ; US, ultrasonography; FNAC, fine-needle aspiration cytology

Key words: fibroadenoma, lobular carcinoma in situ cancer. The present describes a case of LCIS arising within an FA and conducts a review of the relevant literature.

\section{Introduction}

Fibroadenoma (FA) is a common disease of the breast in females. It may occur at any age, however, the peak age of incidence is in the second and third decade of life. The FA can be stimulated by estrogen, progesterone, pregnancy and lactation, and may undergo atrophy during menopause $(1,2)$. Furthermore, FA is a biphasic tumor composed of stromal and epithelial components that is typically considered to be benign, and can be effectively treated by local excision (3).

The majority FAs are clinically identifiable, however, $25 \%$ of cases are non-palpable and, thus, require mammography and/or ultrasonography (US) for diagnosis (4-6). FAs can be classified as simple or complex according to specific histological features, with the risk of malignant transformation higher in patients with complex FA (7). Malignant transformation within FA is unusual, reported to be $0.1-0.3 \%$ in a screened population. However, the number of real cases appears to be even rarer; histological diagnosis is typically unexpected following excision of a breast lesion, with only $>100$ cases reported in the literature thus far $(8,9)$. Classic lobular carcinoma in situ (LCIS) is a type of lobular neoplasia and is diagnosed when $>50 \%$ of the acini of a lobular unit are distended and distorted by a dyshesive proliferation of cells with small, and uniform nuclei (10).

The present study describes the case of a female that had been followed up for 20 years due to the occurrence of multiple breast lesions; in the current study, the patient presented with lobular carcinoma in situ (LCIS) arising within an FA. In addition, a review of the relevant literature was conducted. As no definitive clinical or radiological criteria exist for diagnosing carcinoma arising in an FA, the current case was presented to increase awareness of this entity and to discuss its management in females of different ages.

\section{Case report}

In February 2009, a 44-year-old female presented to Beijing Hospital (Beijing, China) with lumps in the left and right breasts. Over the past 20 years, numerous lumps were detected in the patient's breasts, all of which were clinically diagnosed 
as FAs and treated with regular follow-up once a year. One year prior to presentation, the patient's father was diagnosed with esophageal cancer, the patient's older sister was diagnosed with kidney cancer and the patient herself with a benign ovarian teratoma. The follow-up was stopped in 2008. In 2009, physical examination identified one enlarged lump in each breast. The largest mass in the left breast (lump A) increased in size from $1.2 \times 1.5 \mathrm{~cm}^{2}$ in December 2007 to $2.9 \times 1.8 \mathrm{~cm}^{2}$ in February 2009, as determined by US. Lump A was a lobular and well-circumscribed heterogeneous echoic mass with a number of hyperechoic spots. The largest mass in the right breast (lump B) increased in size from $1.4 \times 1.1 \mathrm{~cm}^{2}$ in December 2007 to $2.7 \times 2.0 \mathrm{~cm}^{2}$ in February 2009. In addition, lump B was determined to be a regular and well-circumscribed homogeneous echoic mass. Doppler US identified a number of arterial vascularities around lump A (Fig. 1). Furthermore, the mammography revealed two high-density lobular-shaped masses in the left breast with round, scattered calcification inside, and two high-density regularly-shaped masses with a clear margin in the right breast (Fig. 2). Fine-needle aspiration cytology (FNAC) determined a diagnosis of benign FA in the two lumps.

On February 13, 2009, the largest masses (lumps A and B) were surgically excised. Lump A was histologically diagnosed as LCIS, therefore, a mastectomy was performed on the left breast. Subsequently, lumpectomies were performed on the remaining masses in the right breast.

Lump A was a hard, elastic and lobular mass, measuring $2.8 \times 2.0 \times 2.0 \mathrm{~cm}^{3}$ (Fig. 3). A jelly-like lesion within the mass was observed the cut surface, measuring $5 \mathrm{~mm}$ in diameter and exhibiting a well-defined boundary. Histological examination confirmed a diagnosis of low grade LCIS within an FA (Fig. 4). Lump B comprised two discrete lesions with an overall size of $2.6 \times 2.5 \times 2.0 \mathrm{~cm}^{3}$ (Fig. 3). Each cut surface was well-circumscribed, solid and greyish in color. Histological examination confirmed the diagnosis of FA.

Postoperatively, the patient received endocrine therapy with tamoxifen, as the LCIS was determined to be ER and PR positive. Clinic visits, including US, occurred every six months, and mammographies were performed every two years. After a five-year follow-up, no local recurrence or contralateral breast cancer were observed. Written informed consent was obtained from the patient for publication of this case report and any accompanying images. The study was approved by the ethics committee of Beijing Hospital.

\section{Discussion}

FA is a common type of benign breast lesion with a reported incidence rate of $7-13 \%$ in female individuals. FA has been reported from adolescence to the middle of the second decade of life in breast disease clinics and occurs asymptomatically in $25 \%$ of all patients. Furthermore, $13-20 \%$ of patients exhibit multiple FAs $(4-6,11)$. According to its clinicopathological presentation, FA can be divided into simple and complex FA. Complex FA is characterized by cysts, sclerosing adenosis, epithelial calcification or papillary apocrine change $(1,11,12)$.

A carcinoma arising within an FA is uncommon, with an incidence rate of $0.1-0.3 \%$ in a screened population (10).
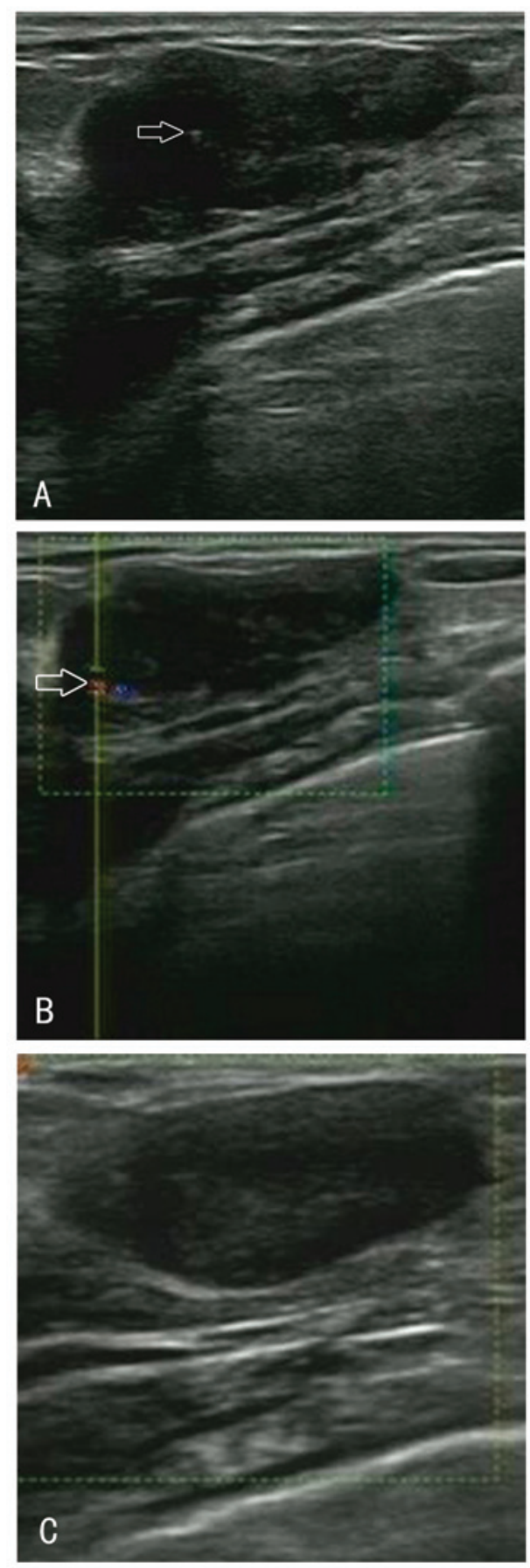

Figure 1. (A and B) Doppler ultrasonography (US) of lump A on US, revealing a lobular and well-circumscribed heterogeneous echoic mass with (A) a number of hyperechoic spots inside it (white arrow) and (B) increasing arterial vascularity around the lesions (white arrow). (C) US of lump B on US, revealing a regular and well-circumscribed homogeneous echoic mass.

However, the actual number of cases is even rarer, with only $>100$ cases reported in the worldwide literature thus far. Therefore, histological diagnosis is typically unexpected $(1,8,9,13,14)$. The mean age of patients with carcinoma in FA has been reported as 42-44 years, $\sim 20$ years subsequent to the peak age of occurrence of FA $(15,16)$. Two-thirds of carcinomas within FA are lobular, and one-third are ductal or mixed ductal and lobular, with the frequency of LCIS and ductal carcinoma in situ almost equal $(2,15)$. Carcinoma is 

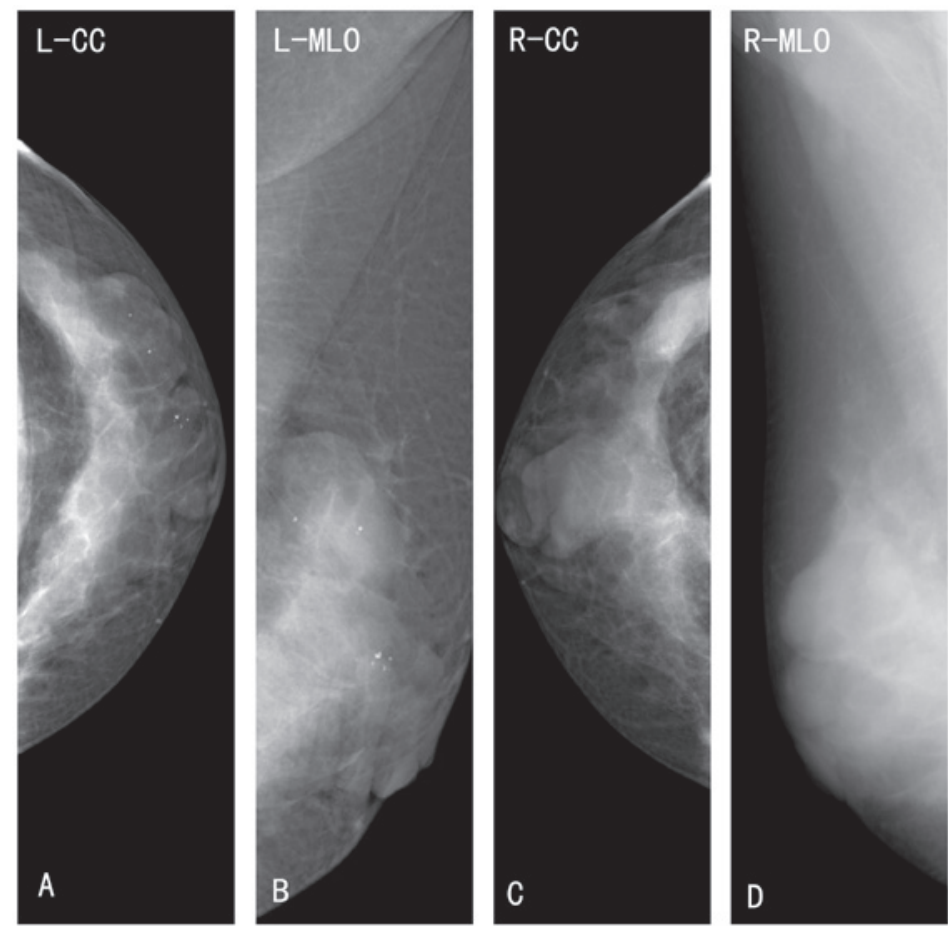

Figure 2. (A and B) Mammography identified two well-defined lobular masses in the left breast with round containing scattered calcification. (C and D) Additionally, mammography revealed two high-density regularly-shaped masses with a clear margin in the right breast. L-CC, left-cranio-caudal; L-MLO, left-mediolateral oblique; R-CC, right-cranio-caudal; R-MLO, right-mediolateral oblique.
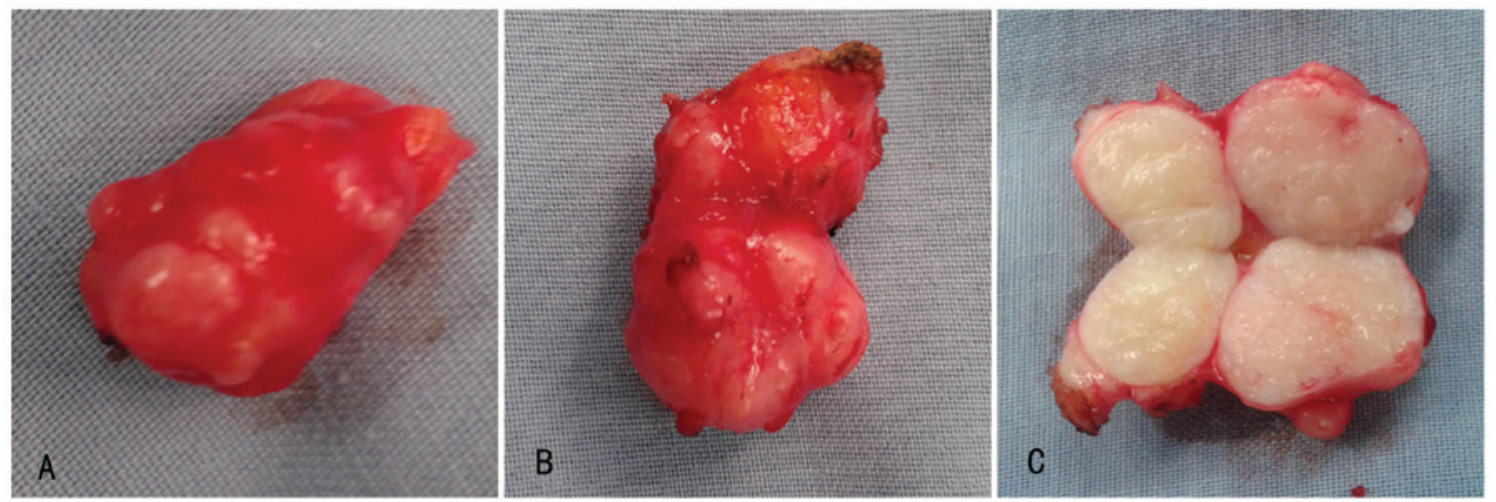

Figure 3. Biopsy specimens. (A) Lump A is a lobular mass; and (B and C) lump B consists of two discrete masses.
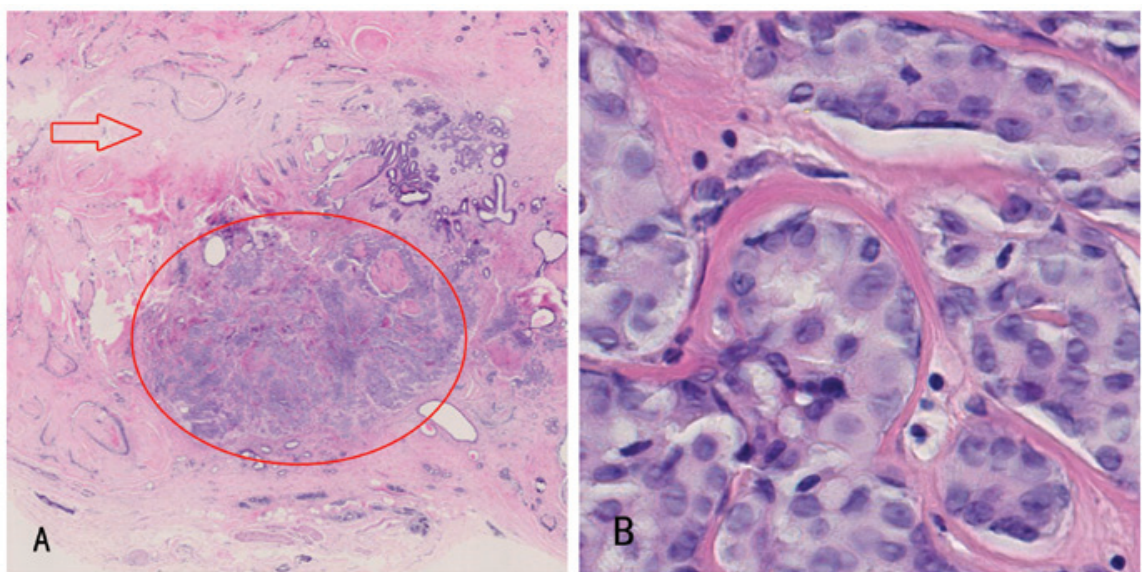

Figure 4. Histological examination using hematoxylin and eosin staining revealed (A) nests of lobular carcinoma in situ (LCIS; red circle) in the center of a typical fibroadenoma (red arrow; magnification, x5). (B) Nests of LCIS (magnification, x40). (B) Nests of LCIS (magnification, x40): the characteristic cells distend and distort the acini. 
diagnosed as arising within FA if: i) The carcinoma is entirely encased within the FA; or ii) if the carcinoma is only focally involved with the adjacent breast tissue (3). The case presented in the present study met the first criterion.

FA is typically considered to be a type of benign lesion and requires careful observation. However, data regarding whether FA is a risk factor for carcinoma is inconsistent (17). A number of studies have provided evidence of the association between the excised FA and carcinoma. The relative risk of developing breast cancer following surgically excised FA appears to depend on the histological characteristics of FA and the state of the breast parenchyma $(18,19)$. Dupont et al $(18)$ identified that patients with benign proliferative disease in the parenchyma adjacent to the FA had a relative risk of developing carcinoma of 3.88 (95\% CI, 2.1-7.3) and patients with complex FA had a relative risk of 3.10 (95\% CI, 1.9-5.1). This risk remained elevated for decades after diagnosis. By contrast, two-thirds of the patients with simple FA and with no family history of breast cancer did not exhibit an increased risk of carcinoma. However, the aforementioned studies do not explain the association between non-excised FA and the occurrence of carcinoma.

Reports of malignant breast neoplasms are common in the literature; however, few studies exist regarding benign breast neoplasms. Therefore, management of FA, in particular the management of multiple FAs, is often a dilemma for surgeons. FA is considered to be an aberration of normal breast development (20) and the majority that occur in younger females may resolve or reduce in size without intervention $(6,21)$. Although malignancy in FA is rare it may be difficult to predict malignant transformation in an FA, as the clinical and radiological signs may be masked $(2,22)$. There is a general consensus that the management of FA depends on the age of the patient, the nature of the lesion, the family history of breast or ovary carcinoma, or any data of proliferative changes in the breasts from previous biopsies $(6,21)$. For example, previous studies have proposed a conservative therapeutic strategy for FA in patients younger than a certain age. However, the threshold ages proposed or conservative treatment are inconsistent, and have been reported as 25, 35 and 45 years (23-26).

At the breast clinic of Beijing Hospital (Ministry of Health, Beijing, China), >20,000 patients are examined each year. Over $75 \%$ of biopsies of benign breast lesions are diagnosed as FA. At the breast clinic of Beijing Hospital, the majority of lesions can be diagnosed by performing the following triple assessment: i) A clinical examination; ii) FNAC or core needle biopsy; and iii) imaging methods, for example US and/or mammography (if the patient is $>35$ years of age or a malignant diagnosis is suspected) or magnetic resonance imaging. If all the results are consistent with simple FA, two different treatment approaches are recommended, dependent on whether the patient is younger or older than the cut-off age of 35 years. For females aged $<35$ years, expectant management with a protocol of follow-up every six months is recommended in order to detect any changes in the lesion. If the lesion increases in size, dissection is immediately performed. However, if the lesion is regressive, follow up should be continued until the regression is complete. If the lesion does not completely regress, or stabilizes by the age of 35 years, a lumpectomy is suggested. For females aged $>35$ years, surgery is recommended when the lesion is stable or increases in size after a 6- to 12-month follow-up period. Additionally, excision is recommended for any complex FA. For patients with a family history of breast and/or ovarian cancer, surgery is more actively advised. Furthermore, the recommendations for the treatment of multiple FAs is the same as that for single FA, and dissection is advised for any lesions with an unclear diagnosis. Patients have the right to refuse surgery.

In conclusion, the present case was presented to increase awareness of this entity and to discuss its management in females of different ages.

\section{References}

1. Kuijper A, Mommers EC, van der Wall E and van Diest PJ: Histopathology of the fibroadenomas of the breast. Am J Clin Pathol 115: 736-742, 2001.

2. Kuijper A, Preisler-Adams SS, Rahusen FD, et al: Multiple fibroadenomas harboring carcinoma in situ in a woman with a family history of breast/ovarian cancer. J Clin Pathol 55: 795-797, 2002.

3. Azzopardi JG, Ahmed and Mills RR: Problems in breast pathology. In: Major Problems in Pathology. Bennington JC (ed). WB Saunders Company Ltd, Edinburgh, pp325-328, 1979.

4. Drukker BH: Breast disease: a primer on diagnosis and management. Int J Fertil Womens Med 42: 278-287, 1997.

5. Foster ME, Garrahan N and Williams S: Fibroadenoma of the breast: a clinical and pathological study. J R Coll Surg Edinb 33: 16-19, 1988.

6. Greenberg R, Skornick Y and Kaplan O: Management of breast fibroadenomas. J Gen Intern Med 13: 640-645, 1998.

7. Buzanowski-Konakry K, Harrison EG Jr and Payne WS: Lobular carcinoma arising in fibroadenomas of the breast. Cancer 35: 450-456, 1975.

8. Iyengar KR, Peh SC, Yip CH and Vijayananthan A: Infiltrating duct carcinoma within a fibroadenoma. Indian J Cancer 46: 244-246, 2009.

9. Abe H, Hanasawa $\mathrm{K}$, Naitoh $\mathrm{H}$, et al: Invasive ductal carcinoma within a fibroadenoma of the breast. Int J Clin Oncol 9: 334-338, 2004.

10. Lakhani SR, Ellis LO, Schnitt SJ , et al: WHO classification of tumours of the breast. In: Lobular neoplasia. Lakhani SR, Schnitt SJ, Malley FO, van de Vijver MJ, Simpson PT and Palacios J (eds). IARC Press, Lyon, pp77-80, 2012.

11. Dixon JM and Mansel RE: ABC of breast diseases. Congenital problems and aberrations of normal breast development and involution. BMJ 309: 797-800, 1994.

12. Tissier F, De Roquancourt A, Astier B, Espie M, Clot P, Marty $M$ and Janin A: Carcinoma arising within mammary fibroadenomas. A study of six patients. Ann Pathol 20: 110-114, 2000 (In French).

13. Sklair-Levy M, Sella T, Alweiss T, Craciun I, Libson E and Mally B: Incidence and management of complex fibroadenomas. AJR Am J Roentgenol 190: 214-218, 2008.

14. Netto D, Satchidanand SK and Gaeta JF: Carcinomas arising in fibroadenomas: a report of two cases and a review of literature. J Surg Oncol 13: 367-372, 1980.

15. Diaz NM, Palmer JO and McDivitt RW: Carcinoma arising within fibroadenomas of the breast. A clinicopathologic study of 105 patients. Am J Clin Pathol 95: 614-622, 1991.

16. Pick PW and Iossifides IA: Occurrence of breast carcinoma within a fibroadenoma. A review. Arch Pathol Lab Med 108: 590-594, 1984

17. Dent DM and Cant PJ: Fibroadenoma. World J Surg 13: 706-710, 1989.

18. Dupont WD, Page DL, Parl FF, Vnencak-Jones CL, Plummer WD Jr, Rados MS and Schuyler PA: Long-term risk of breast cancer in women with fibroadenoma. N Engl J Med 331: 10-15, 1994.

19. McDivitt RW, Stevens JA, Lee NC, Wingo PA, Rubin GL and Gersell D: Histologic types of benign breast disease and the risk for breast cancer. The Cancer and Steroid Hormone Study Group. Cancer 69: 1408-1414, 1992.

20. Hughes LE, Mansel RE and Webster DJ: Aberrations of normal development and involution (ANDI): a new perspective on pathogenesis and nomenclature of benign breast disorders. Lancet 2: 1316-1319, 1987. 
21. Carty NJ, Carter C, Rubin C, Ravichandran D, Royle GT and Taylor I: Management of fibroadenoma of the breast. Ann R Coll Surg Engl 77: 127-130, 1995.

22. Goldman RL and Friedman NB: Carcinoma of the breast arising in fibroadenoma, with emphasis on lobular carcinoma. A clinicopathologic study. Cancer 23: 544-550, 1969.

23. Cant PJ, Madden MV, Close PM, Learmonth GM, Hacking EA and Dent DM: Case for conservative management of selected fibro-adenomas of the breast: Br J Surg 74: 857-859, 1987.
24. Sainsbury JR, Nicholson S, Needham GK, Wadehra V and Farndon JR: Natural history of the benign breast lump. Br J Surg 75: 1080-1082, 1988.

25. Wilkinson S, Anderson TJ, Rifkind E, Chetty U and Forrest AP: Fibroadenoma of the breast: a follow-up of conservative management. Br J Surg 76: 390-391, 1989.

26. Dixon JM, Dobie V, Lamb J, Walsh JS and Chetty U: Assessment of the acceptability of conservative management of fibroadenoma of the breast. Br J Surg 83: 264-265, 1996. 